\title{
Correlative Electron Microscopy Enables Scalable Characterization of 2D half-van der Waals Heterostructures
}

Hesham El-Sherif ${ }^{1}$, Natalie Briggs ${ }^{2}$, Joshua Robinson ${ }^{2}$ and Nabil Bassim ${ }^{3}$

${ }^{1}$ McMaster University, Hamilton, Ontario, Canada, ${ }^{2}$ The Pennsylvania State University, State College, Pennsylvania, United States, ${ }^{3}$ Department of Materials Science and Engineering, McMaster University, Hamilton, Ontario, Canada, Hamilton, Ontario, Canada

The confinement heteroepitaxy (CHet) technique has recently been used for the scalable synthesis of van der Waals heterostructures [1]. In this process, atoms intercalate the interface between epitaxial graphene (EG) and silicon carbide ( $\mathrm{SiC}$ ) substrates, facilitating metastable 2D nitrides, 2D oxides, and 2D metals that are environmentally protected by the EG over vast scalable areas. 2D Gallium (2D Ga) and 2D Indium (2D In) - which are characterized as half-van der Waal 2D metal heterostructures because of the bonding difference across the interface - are ones of the newly advanced 2D metals [1]. Interestingly, exotic optical and electronic properties are exhibited in 2D Ga and 2D, such as huge nonlinear optical response [2], superconductivity [1], and near-zero index [3]. In prior work, large-area spectroscopic photon-based techniques - such as XPS and spectroscopic ellipsometry - report the coverage of the 2D Ga and 2D In on the $\mathrm{SiC}$ substrate $[1,3]$. However, the area coverage of these heterostructures is not quantitatively understood with high spatial resolution techniques yet. Here, we use the contrast from scanning electron microscope (SEM) images to unlock the metal intercalation positions as well as the EG thicknesses. We applied multiple correlative electron microscopy techniques for understanding the secondary electron (SE) emission that generates the contrast in SEM images. Our correlative electron microscopy includes surface chemical maps in plan view using auger electron spectroscopy (AES) and with Scanning-Transmission electron microscopy (STEM) imaging and spectroscopy obtained from multiple site-specific crosssections (Figure 1). We also understand the origin of the SE emission related to the heterostructure's local work function by correlating both experimental measurements and theoretical calculations of the surface potential of these heterostructures. We also optimized the SEM imaging conditions - current, voltage, contrast/brightness, and the SE detector - to efficiently augment the differential SEM contrasts. Locating the 2D metal heterostructure positions enables us to segment SEM images that cover few millimeters in area. This segmentation process is done manually from the image histogram as well as in an un-supervised manner using machine learning segmentation techniques. We form a stitched montage view of $\sim 2 \mathrm{~mm} x$ $1.5 \mathrm{~mm}$ area by stitching low-magnification and high-pixelated SEM images (Figure 2). Unlike photonbased spectroscopic techniques, The SEM method retains lateral resolution controlled by the SEM resolution (few $\mathrm{nm}$ ) while also retaining, as resolved by correlative microscopy, the atomic resolution of the thicknesses of the heterostructure. The SEM spectroscopic imaging allows high-spatial-resolution for tracking intercalants, identify relative surface potentials, or determining the number of 2D layers and heterostructures. This could be further employed to characterize the uniformity and quality of Chet-grown 2D materials and 2D Van der Waals heterostructures at wafer scale. 

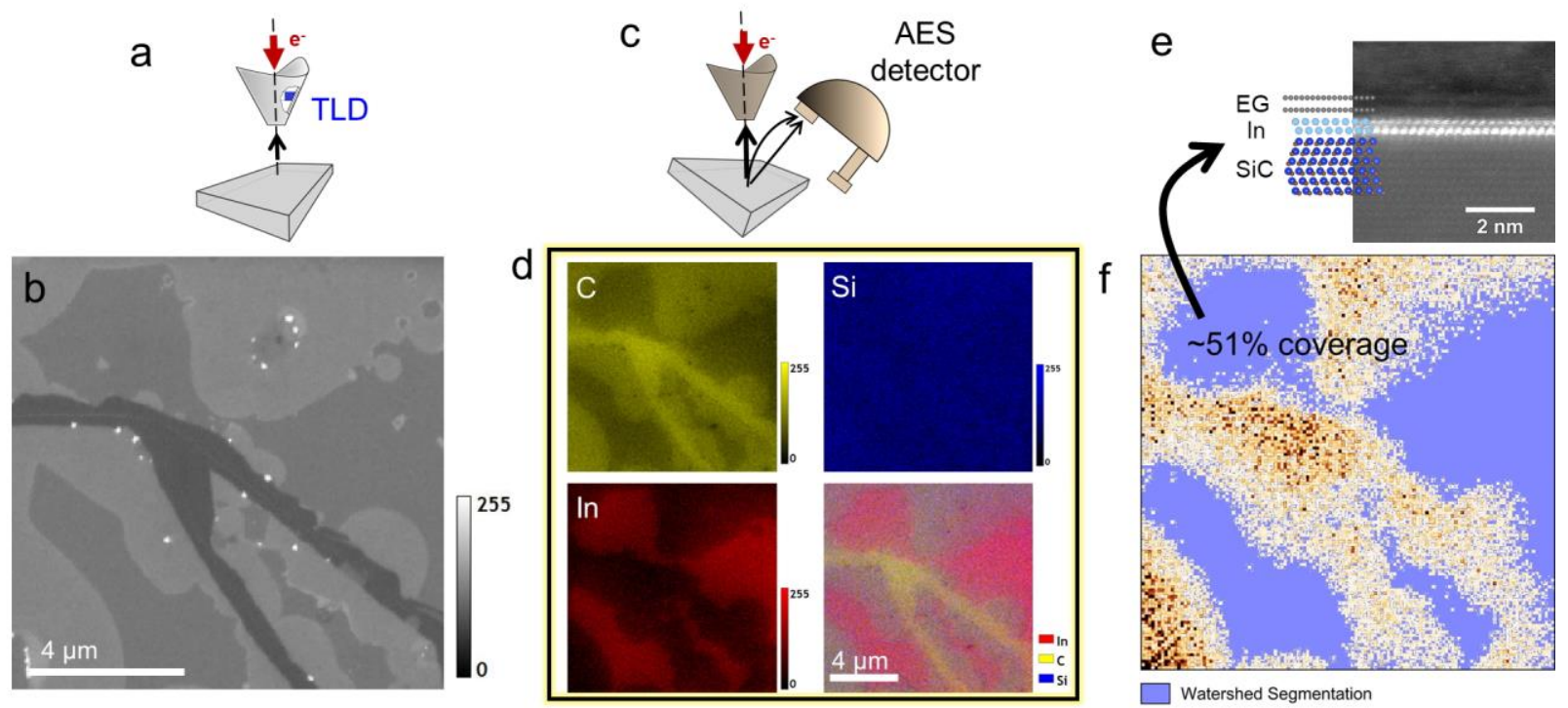

Figure 1. Figure 1. Correlative Electron Microscopy of 2D Indium (2D In) heterostructure. (a) illustration figure of the SEM imaging coordination using a through-lens detector (TLD). (b) SEM image of the 2D In heterostructure. (c) illustration figure of using auger electron spectroscopy (AES) for chemical mapping the surface. (d) AES elemental mapping of the same area on (b). (e) STEM-HAADF image of the 2D In heterostructure with an atomic model in the left. (f) a watershed segmentation of the AES indium map in the (d) presenting a $\sim 51 \%$ coverage.
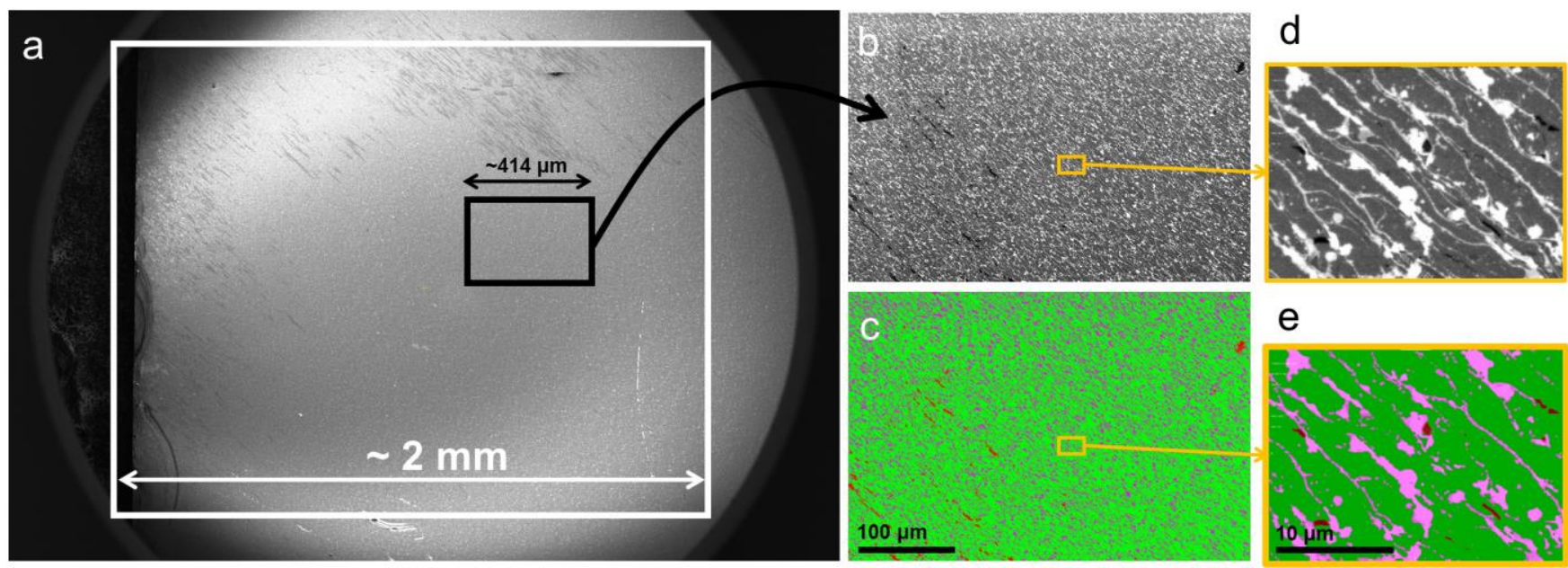

Figure 2. Figure 2. Scalable characterization of 2D Gallium (2D Ga) heterostructure. (a) lowmagnification SEM image coverages a $\sim 2 \mathrm{~mm}$ wide area. (b) and (c) are medium magnification SEM images of the 2D Ga surface and the corresponding segmentation. (d) and (e) are a close-up view from (b) and (c), respectively. Segmentation and coverage calculations are performed on a (5x6) image-matrix that covers all the white box in (a) with a total area of $1.5 \mathrm{~mm} \times 2 \mathrm{~mm}$. 


\section{References}

[1] N. Briggs, B. Bersch, Y. Wang, J. Jiang, R. J. Koch, N. Nayir, K. Wang, M. Kolmer, W. Ko, A. De La Fuente Duran, S. Subramanian, C. Dong, J. Shallenberger, M. Fu, Q. Zou, Y. W. Chuang, Z. Gai, A. P. Li, A. Bostwick, C. Jozwiak, C. Z. Chang, E. Rotenberg, J. Zhu, A. C. T. van Duin, V. Crespi, J. A. Robinson, Nature Materials 2020, 19, 637.

[2] M. A. Steves, Y. Wang, N. Briggs, T. Zhao, H. El-Sherif, B. M. Bersch, S. Subramanian, C. Dong, T. Bowen, A. D. L. Fuente Duran, K. Nisi, M. Lassaunière, U. Wurstbauer, N. D. Bassim, J. Fonseca, J. T. Robinson, V. H. Crespi, J. Robinson, K. L. Knappenberger Jr, Nano Letters 2020, 20, 8312.

[3] K. Nisi, S. Subramanian, W. He, K. A. Ulman, H. El-Sherif, F. Sigger, M. Lassaunière, M. T. Wetherington, N. Briggs, J. Gray, A. W. Holleitner, N. Bassim, S. Y. Quek, J. A. Robinson, U.

Wurstbauer, Advanced Functional Materials 2020, 2005977. 